\title{
Étude des composés organiques volatils biogéniques émis par une forêt méditerranéenne
}

\section{Résumé}

Les composés organiques volatils (COV) sont des composants clés en chimie atmosphérique. À l'échelle globale, on estime que $10 \%$ ont une origine anthropique et $90 \%$ une origine biogénique. Limpact des $\mathrm{COV}$ biogéniques (COVB), notamment sur la formation d'ozone (polluant et gaz à effet de serre) et d'aérosols organiques secondaires (à impact sanitaire et radiatif) en font un objet d'étude d'actualité. Toutefois, l'importance de l'impact des COVB sur la qualité de l'air est encore marquée par de larges incertitudes, en partie parce que les émissions demeurent mal quantifiées. Dans le cadre du projet Canopee, une campagne de mesure a eu lieu à l'O3HP, Observatoire du chêne pubescent à l'OHP (Observatoire de Haute-Provence), avec pour objectif de quantifier les émissions de COVB issues d'une forêt représentative de la région méditerranéenne. Des mesures de concentrations et de flux d'émission ont été réalisées à plusieurs échelles : de la branche à la canopée. L'étude des émissions à l'échelle du de la plante a permis une meilleure compréhension des paramètres environnementaux et physiologiques qui régissent les émissions. Les flux de COVB sortant de la canopée et exportés vers la troposphère ont pu être quantifiés. Les résultats montrent que le chêne blanc est un fort émetteur d'isoprène, mais un faible émetteur de méthanol et de monoterpènes. À l'échelle de la forêt, les flux d'émissions d'isoprène ont été estimés à $7,2 \mathrm{mg} \mathrm{m}^{-2} \mathrm{~h}^{-1}$ en conditions standard, ce qui fait de l'O3HP un des écosystèmes les plus fortement émetteurs d'isoprène à travers le monde.

\author{
Athina-Cerise Kalogridis ${ }^{*}$, Valérie Gros', Bernard Bonsang1, \\ Roland Sarda-Esteve ${ }^{1}$, Anne-Cyrielle Genard1,2, Christophe Boissard1, \\ Catherine Fernandez², Elena Ormeño², Nicolas Bonnaire', \\ Dominique Baisnée ${ }^{1}$, Juliette Lathière \\ 1 Laboratoire des sciences du climat et de l'environnement, CEA / CNRS / \\ Université de Versailles Saint-Quentin-en-Yvelines, Gif-sur-Yvette \\ 2 Institut méditerranéen de la biodiversité et d'écologie marine \\ et continentale, Université d'Aix-Marseille / CNRS / IRD / Avignon \\ Université, Marseille
}

akalogridi@ipta.demokritos.gr

a biosphère terrestre joue un rôle essentiel dans le cycle global du carbone. Bien que les échanges gazeux des plantes soient dominés par l'émission et l'absorption de dioxyde de carbone, vapeur d'eau et oxygène, les plantes produisent et émettent également une autre classe de composés : les composés organiques volatils biogéniques (COVB). La famille des composés organiques volatils regroupe plusieurs milliers de composés aux caractéristiques chimiques très diverses, mais qui ont en commun de posséder les éléments carbone, oxygène et hydrogène, mais aussi d'autres éléments parmi les halogènes ou encore le soufre. Comme leur nom l'indique, ces composés peuvent se trouver facilement sous forme gazeuse et se disperser dans l'atmosphère. Plus de 5000 molécules chimiques volatiles émises par les plantes (Steiner et Goldstein, 2008) sont connues à ce jour, mais seules quelques-unes d'entre elles contribuent de manière significative aux flux de COV dans l'atmosphère à l'échelle du globe.

\section{Parmi les COV, la famille des isoprénoïdes}

La production de COV par la végétation est dominée par les émissions d'isoprénoïdes, famille de composés hydrocarbonés, pouvant être cycliques ou à chaîne ouverte, ayant pour formule brute $\left(\mathrm{C}_{5} \mathrm{H}_{8}\right)_{n}$. L'isoprène $(n=1)$ est le composé le plus volatil de cette famille et aussi le plus abondamment émis par les écosystèmes à l'échelle globale. À lui seul, il représente environ $50 \%$ des émissions globales de COVB. Il est essentiellement produit par les arbres à feuilles caduques et son taux d'émission dans l'atmosphère varie selon les estimations entre 400 et $600 \mathrm{TgC} \mathrm{an}^{-1}$ (Guenther et al., 2006) sachant que $1 \mathrm{TgC}=10^{12} \mathrm{~g}$ de carbone.

* Les données présentées dans cet article font l'objet d'une publication intitulée Concentrations and fluxes of isoprene and oxygenated VOCs at a French Mediterranean oak forest (Kalogridis et al., 2014).

A.-C. Kalogridis est actuellement au Laboratoire de radioactivité environnementale, NCSR Demokritos, Agia Paraskevi, Grèce. 


\section{Abstract}

\section{Investigating biogenic volatile organic compounds emitted by a Mediterranean forest}

Volatile organic compounds (VOCs) are key components in atmospheric chemistry. On a global scale, biogenic VOCs (BVOCs) constitute approximately $90 \%$ of global VOC emissions. They participate in photochemical reactions and thus play a major role in the formation of tropospheric ozone and secondary organic aerosols. However, the impact of BVOCs on air quality is still characterized by large uncertainties, partly because emissions are not well quantified. As part of the Canopee project, a field campaign took place at the Oak Observatory of the Observatoire de Haute-Provence (O3HP), with the aim of quantifying BVOC emissions from a forest representative of the Mediterranean region. Measurements of concentrations and emission fluxes were carried out from the branch to the canopy level. Branch-level measurements enabled a better understanding of the environmental and physiological parameters of the plant that govern emissions. Flux measurements above the top of the canopy enabled us to quantify the outgoing flow of BVOCs from the forest entering the troposphere. The results showed that white oak is a strong emitter of isoprene but a weak emitter of methanol and monoterpenes. Isoprene emission fluxes were estimated at $7.2 \mathrm{mg} \mathrm{m}^{-2} \mathrm{~h}^{-1}$ (under standard conditions of temperature and solar radiation), thus making the $\mathrm{O3HP}$ forest one of the strongest isoprene emitting ecosystems worldwide.
Les monoterpènes $(n=2)$ sont, quant à eux, essentiellement produits par les résineux. Ce sont des molécules aromatiques responsables des odeurs des plantes. Par exemple, l'odeur caractéristique des agrumes provient de monoterpènes appelés limonènes. Les sesquiterpènes $(n=3)$ sont une composante majeure des huiles essentielles stockées par certaines plantes, en particulier les arbres à feuilles larges. Ils font partie des COVB ayant été les moins étudiés à ce jour, du fait de leur découverte plus récente, et des difficultés de leur détermination analytique. Parmi les autres COVB, on distingue divers composés oxygénés dont les alcools, aldéhydes, esters et acides organiques.

Les taux d'émission des COVB sont très variables selon les espèces et dépendent de paramètres biotiques (métabolisme, physiologie de la plante...) et abiotiques (température, intensité du rayonnement solaire, humidité et autres paramètres physico-chimiques). Ces COVB participent aux régulations allélopathiques que met en place la plante avec son environnement, par exemple pour attirer les insectes pollinisateurs ou encore pour se défendre de prédateurs. Ils peuvent notamment résulter d'un stress subi par la plante (chaleur, sécheresse, présence de polluants tels que l'ozone). Toutefois, de grandes incertitudes demeurent encore quant à leur mécanisme de synthèse et au rôle écologique de leur production. Les émissions de COVB à l'échelle globale sont estimées à environ $1000 \mathrm{Tg} \mathrm{an}{ }^{-1}$ (Guenther et al., 2012), dont environ $71 \%$ proviennent des arbres, $17 \%$ des arbustes, $10 \%$ des cultures et 0,4\% des océans (Guenther et al., 1995). Ces estimations reposent sur des données expérimentales qui servent de paramètres importants dans les outils de modélisation. Néanmoins, ces données de « terrain » sont sporadiques et ne peuvent pas facilement être extrapolées à l'ensemble du globe ; les estimations de flux qui en résultent comportent par conséquent de fortes incertitudes.

\section{Rôle des COV biogéniques en chimie atmosphérique}

Parmi les molécules biogéniques, nombreuses sont celles constituées d'au moins une double liaison oléfinique ${ }^{1}$ qui les rend extrêmement réactives.
Ainsi, en dépit de leurs concentrations atmosphériques relativement faibles, ce sont des éléments clés de la chimie troposphérique. Ces molécules réagissent rapidement avec les oxydants tels que les radicaux hydroxyles $\mathrm{OH}$ et influencent ainsi sensiblement la capacité oxydante de l'atmosphère (Mégie, 1996), et donc la durée de vie des composés gazeux comme le méthane, gaz à effet de serre notoire (Chiemchaisri et al., 2001 ; Wuebbles et al., 1989).

Les COVB jouent également un rôle clé dans le cycle de l'ozone troposphérique. En présence de concentrations suffisamment élevées en oxydes d'azote $\left(\mathrm{NO}_{x}\right)$ et de lumière, les COVB peuvent être d'importants précurseurs d'ozone (Chameides et al., 1988 ; Curci et al., 2010 ; Jacob et Wofsy, 1988 ; Lee et al., 2006). Les émissions de COVB étant fortement dépendantes de la température et de l'ensoleillement, ces composés jouent un rôle parfois significatif dans les niveaux d'ozone atteints en été, lors des épisodes de pollution pendant les périodes anticycloniques, dans des zones péri-urbaines à proximité de sites forestiers importants. Une étude récente estime que les émissions de COVB sont à l'origine d'une augmentation de 5 à 15 ppbv $^{2}$ (jusqu'à 20 ppbv en 2003) des niveaux d'ozone troposphérique dans le bassin méditerranéen en été (Curci et al., 2010).

À partir de scénarios futurs, Lathière et al. (2005) ont estimé une augmentation globale de $70 \%$ des émissions de COVB en 2100 par rapport à nos jours, en prenant en compte l'impact des changements de climat et de $\mathrm{CO}_{2}$ atmosphérique. En incluant ces émissions futures dans un modèle global de chimie-transport, Hauglustaine et al. (2005) ont calculé une augmentation de l'ozone de surface de 30 à $50 \%$ en été dans les régions continentales de l'hémisphère Nord. Par ailleurs, plusieurs études ont démontré que l'oxydation des monoterpènes $\left(\mathrm{C}_{10} \mathrm{H}_{16}\right)$, des sesquiterpènes $\left(\mathrm{C}_{15} \mathrm{H}_{24}\right)$ et, dans une moindre mesure, de l'isoprène, contribuait à la formation d'aérosols organiques secondaires dans la troposphère (Claeys et al., 2004 ; Griffin et al., 1999); de fortes incertitudes demeurent cependant quant à la compréhension et la quantification de ces phénomènes. L'ensemble de ces

1. Liaison oléfinique : double liaison $C=C$.

2. ppbv: partie par milliard sur une base volumique. 1 ppbv du composé $x=1$ volume de $x$ dans $10^{9}$ volumes du mélange gazeux. 
éléments justifie qu'on étudie en particulier les COVB afin de mieux comprendre les processus qui contrôlent la qualité de l'air et l'évolution du climat.

\section{De l'échelle de la feuille à celle du globe}

Depuis les années 1960, de nombreuses équipes scientifiques œuvrent à mesurer les flux d'émission et les concentrations des COVB à différentes échelles spatiales et temporelles, à partir de diverses plateformes instrumentales. À l'échelle de la plante, des études en milieu contrôlé permettent de quantifier de manière précise les taux d'émissions de COVB par quantité de biomasse. Toutefois, ces études ne peuvent être appliquées que sur un nombre très limité d'espèces en raison de la complexité du système expérimental. Les mesures dans l'air libre (au-dessus de forêts, champs, etc.) intègrent quant à elles les émissions d'un ensemble de végétaux constituant un écosystème. Enfin, des mesures aéroportées permettent d'étudier la chimie et le transport atmosphérique des COVB à des échelles spatiales encore plus larges. L'ensemble de ces données peut ensuite être intégré dans divers outils de modélisation numérique afin d'estimer les émissions de COVB à l'échelle globale, le devenir de ces émissions, ainsi que leur impact sur la qualité de l'air et le climat.

\section{Mesure des concentrations atmosphériques}

Pour la mesure des COV dans l'air, on distingue les méthodes indirectes (off-line) des méthodes directes (on-line). Dans les méthodes indirectes, les échantillons gazeux sont analysés en différé au laboratoire. Les méthodes directes permettent, quant à elles, le prélèvement et l'analyse simultanés d'échantillons d'air. Elles présentent donc l'avantage de fournir des données sur le terrain, avec une meilleure résolution temporelle et sans artefact lié à la conservation de l'échantillon. Parmi les nombreuses méthodes on-line, on distingue un outil novateur, sensible et rapide : le spectromètre de masse par réaction de transfert de proton (PTR-MS ou proton transfer reaction-mass spectrometry). Cette nouvelle technologie a ouvert la voie à de nombreux développements dans la recherche sur les émissions de COVB (de Gouw et Warneke, 2007). Ne nécessitant aucune préparation de l'échantillon préalable à l'analyse, il permet la mesure in situ et en temps réel d'une large gamme de composés (terpènes, aldéhydes, cétones, oxygénés...) simultanément dans l'air, avec une limite de détection d'une dizaine de pptv ${ }^{3}$ pour la plupart des masses mesurées.

\section{Mesure des flux d'émission}

Afin d'étudier les processus d'échange entre la biosphère et l'atmosphère, des techniques de mesure des flux ont été développées. Par convention, on appelle "flux d'émission » un flux vers l'atmosphère et "flux de dépôt " un flux vers la surface. De manière générale, on distingue les méthodes de chambre (couvrant des surfaces naturelles de l'ordre de 0,1 à $1 \mathrm{~m}^{2}$ ) et les méthodes micrométéorologiques, qui permettent d'intégrer les flux sur des surfaces plus grandes, de quelques dizaines de mètres carrés à plusieurs kilomètres carrés.

\section{À l'échelle de la plante}

Les chambres d'enfermement dynamiques permettent d'enfermer un végétal (soit en partie, soit dans son intégralité, selon sa taille) et d'étudier ses échanges gazeux avec l'atmosphère environnante (figure 1). Pour comprendre les processus qui se déroulent à l'intérieur de la chambre (émission ou absorption de gaz), il est nécessaire de déterminer les teneurs des gaz entrant mais également sortant de la chambre. Parmi les composés cibles, la vapeur d'eau et le dioxyde de carbone sont mesurés à chaque instant et vont témoigner des processus photosynthétiques du végétal.

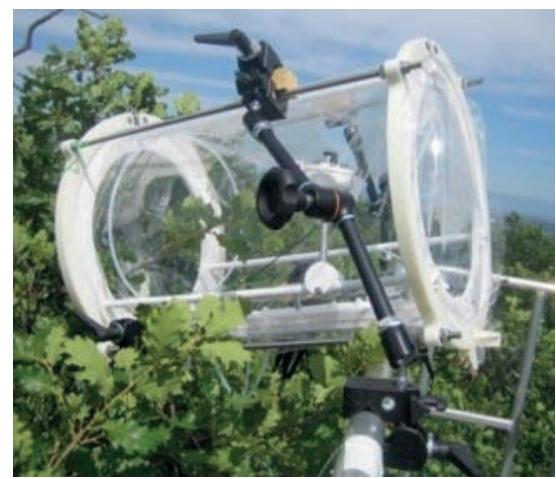

Figure 1. Chambre dynamique en téflon enfermant une branche de chêne blanc au sommet de la canopée.
De même, des prélèvements sur cartouches (ou via un analyseur en ligne) sont effectués afin d'estimer la quantité de COV émis par la plante dans l'enceinte. Les taux d'émission des COVB sont calculés à partir de la différence de concentration de l'espèce gazeuse considérée dans l'air entrant et sortant de la chambre. Un ensemble de capteurs placés dans la chambre permettent de mesurer les conditions (lumière, température, humidité de l'air) dans lesquelles la plante a effectué ces échanges gazeux.

\section{À l'échelle d'un écosystème}

Dans les méthodes micrométéorologiques, le flux turbulent vertical est mesuré au-dessus de la surface (une forêt, par exemple) et à l'intérieur de la couche limite atmosphérique (figure 2). Ces méthodes de mesure présentent l'avantage de ne pas perturber la végétation émettrice et d'être représentatives d'une grande surface. Elles reposent sur le principe que la couche limite atmosphérique est caractérisée par la présence de mouvements turbulents qui sont responsables du transport vertical de certains composés gazeux. Parmi les différentes méthodes micrométéorologiques, la technique d'eddy covariance, appelée en français méthode de covariance des turbulences, est la méthode de référence pour la mesure directe de flux de traceurs entre l'atmosphère et la surface. Son principe consiste à mettre en corrélation deux informations fondamentales : les fluctuations de la vitesse du vent vertical $w$ et de la concentration de l'espèce considérée $c$. Les deux composantes $w$ et $c$ doivent être mesurées simultanément et à haute fréquence $(10-50 \mathrm{~Hz})$ afin de prendre en compte les fluctuations contribuant au flux (Aubinet et al., 2012). Néanmoins, cette méthode de référence ne peut être utilisée que pour un nombre restreint de traceurs (flux de chaleur, de vapeur d'eau, de $\mathrm{CO}_{2}$ ou bien d'ozone), car peu d'analyseurs sont capables de réaliser des mesures à des fréquences suffisantes. Afin d'élargir le champ de mise en œuvre de cette technique, d'autres techniques été développées, dont la méthode de covariance disjointe des turbulences (disjunct eddy covariance), avec laquelle le calcul de la covariance du flux repose sur un nombre réduit de valeurs du scalaire c, permettant ainsi l'utilisation d'analyseurs plus lents (Karl

3. pptv : partie par trillion sur une base volumique. 1 pptv du composé $x=1$ volume de $x$ dans $10^{12}$ volumes du mélange gazeux. 


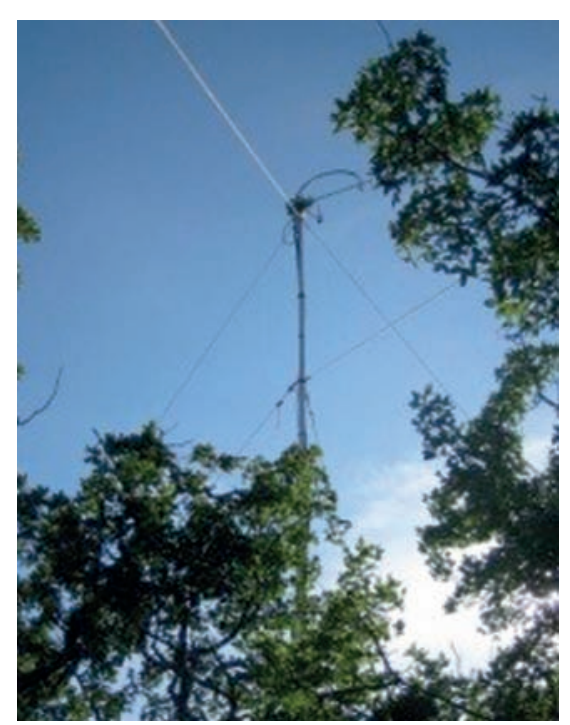

Figure 2. Mât de 10 mètres sur lequel sont fixées les lignes de prélèvement de l'air, ainsi que l'anémomètre sonique pour la mesure du vent.

et al., 2002 ; Rinne et al., 2001 ; Taipale et al., 2010). Cette technique est particulièrement adaptée pour l'étude des flux de COVB au moyen d'un spectromètre de masse par réaction de transfert de proton, et, pour cela, elle a été mise en œuvre pendant la campagne Canopee.

\section{Étude des COVB dans une forêt méditerranéenne de chêne blanc}

\section{Objectif du projet Canopee}

Aujourd'hui, la communauté française travaillant en chimie atmosphérique porte une attention particulière à l'étude du bassin méditerranéen. Le programme international Charmex (Chemistry aerosol mediterranean experiment), développé dans le cadre du métaprogramme Mistrals (Mediterranean integrated studies at regional and local scales), a pour objectif de mieux caractériser la pollution atmosphérique $\mathrm{du}$ bassin méditerranéen et de son pourtour pour en identifier les sources, les facteurs aggravants et les conséquences sur l'environnement à court et long terme. Ce bassin, caractérisé par des températures et des niveaux d'ensoleillement élevés, est sous l'influence de grandes zones

4. https://o3hp.obs-hp.fr urbaines et industrielles, mais aussi de sources biogéniques constituées par de grandes zones de forêt. C'est dans ce contexte que s'inscrit le projet Canopee qui vise à une caractérisation expérimentale fine des COVB en zone émettrice, dans un milieu dont la végétation est représentative des espèces trouvées en zone méditerranéenne. Cette caractérisation inclut une spéciation des molécules émises (isoprène, terpènes, oxygénés) et une évaluation des flux d'émission. Elle permet d'étudier la chimie intracanopée (réactions chimiques et dépôt se déroulant dans la canopée) et cherche à estimer l'export des composés hors canopée pour le milieu étudié.

\section{L'Observatoire du chêne blanc}

L'Observatoire de Haute-Provence (OHP) est un domaine de 95 hectares (figure 3) situé dans le parc naturel du Lubéron, à $15 \mathrm{~km}$ au nord-ouest de Manosque, sur un plateau à $650 \mathrm{~m}$ d'altitude. Le climat du site est méditerranéen, aux étés chauds et secs et aux hivers frais. Propriété du CNRS depuis 1939, l'OHP est en premier lieu un observatoire astronomique et possède également une station d'étude de l'atmosphère. Depuis 2008, une zone protégée de 1'OHP, dénommée O3HP ${ }^{4}$ (Oak Observatory à l'Observatoire de Haute-Provence) est dédiée à l'étude de la dynamique, du fonctionnement et de la biodiversité d'une forêt méditerranéenne (Hilaire et al., 2013). C'est au sein de cette plateforme expérimentale couverte par une forêt de chêne blanc à $~ 90 \%$ et d'érables de Montpellier à $~ 10 \%$ que les campagnes Canopee ont eu lieu.

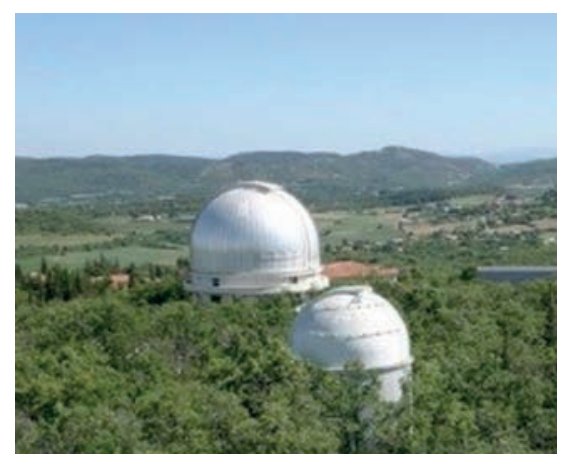

Figure 3. L'Observatoire de Haute-Provence sur la commune de Saint-Michel I'Observatoire, dans les Alpes-de-Haute Provence.

\section{Le chêne blanc, un émetteur d'isoprène}

Le chêne blanc, aussi appelé chêne blanc de Provence, ou chêne pubescent, est un arbre des régions tempérées de l'hémisphère Nord, appartenant à la famille des Fagaceae. Son nom vient du latin pubescens qui signifie à poils courts et mous. Il est marcescent, c'est-à-dire qu'à l'automne ses feuilles sèchent mais restent en place tout l'hiver. Ce n'est qu'à la fin de la saison froide que les dernières feuilles tombent, avec la poussée des jeunes feuilles vertes. Le chêne blanc est une espèce très largement établie en région méditerranéenne. Il recouvre environ 260000 ha en région Paca, soit $20 \%$ de la surface forestière et constitue le principal émetteur d'isoprène en région méditerranéenne. À l'échelle européenne, on estime qu'il est responsable d'environ $15 \%$ des émissions d'isoprène (Keenan et al., 2009).

\section{Stratégie expérimentale}

Un suivi saisonnier des concentrations atmosphériques en isoprène a été réalisé de mai 2011 à mai 2012. Pour cela, l'air a été échantillonné sur cartouches d'adsorbants, une à deux fois par mois pendant 24 heures, puis analysé au laboratoire.

La campagne de mesure intensive Canopee a eu lieu en juin 2012. Des mesures de COV ont été réalisées à différentes échelles : au niveau de la branche et au niveau de la canopée (figure 4). À l'échelle de la plante, les mesures de COVB ont été effectuées à l'aide de chambres d'enfermement dynamiques. Une première branche de chêne blanc située dans la partie basse de la canopée a été étudiée pendant 24 heures. Les mesures par PTR-MS ont permis de suivre, à haute résolution temporelle, un large nombre de composés et d'identifier ceux majoritairement émis par le chêne blanc. Six autres branches, situées sur la partie haute et basse de la canopée, ont été mises à l'étude, afin d'observer la variabilité des taux d'émission d'isoprène entre chaque branche. Les mesures d'isoprène ont été effectuées par prélèvement horaire sur cartouches analysées par chromatographie en phase gazeuse couplée à la spectrométrie de masse (GC-MS).

À l'échelle de la canopée, deux niveaux de mesure ont été choisis : à l'intérieur de la forêt, à $2 \mathrm{~m}$ au-dessus du sol, et à 


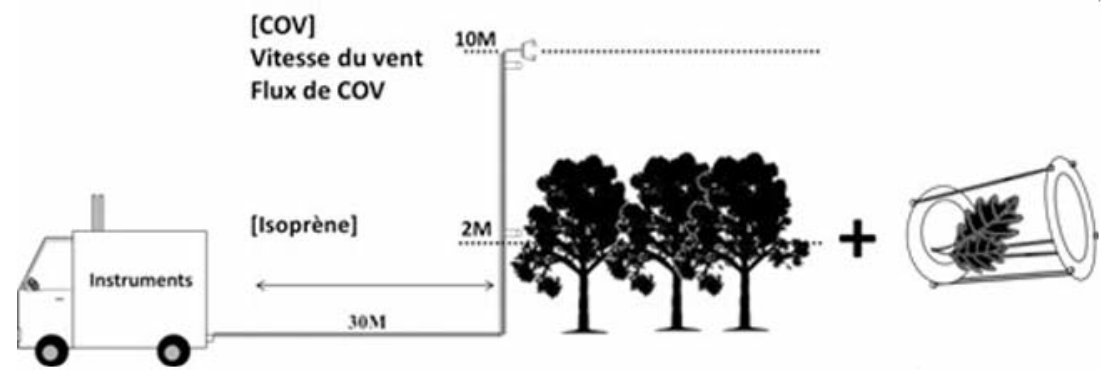

Figure 4. Schéma descriptif de la stratégie expérimentale adoptée pendant la campagne intensive Canopee.

$5 \mathrm{~m}$ au-dessus de la canopée, c'est-à-dire à $10 \mathrm{~m}$ au-dessus du sol. Les lignes de prélèvement de l'air ont été installées sur un mât situé en plein cœur de la forêt et reliées aux appareils analytiques situés dans un camion instrumenté, éloigné du mât afin de minimiser toute perturbation du milieu.
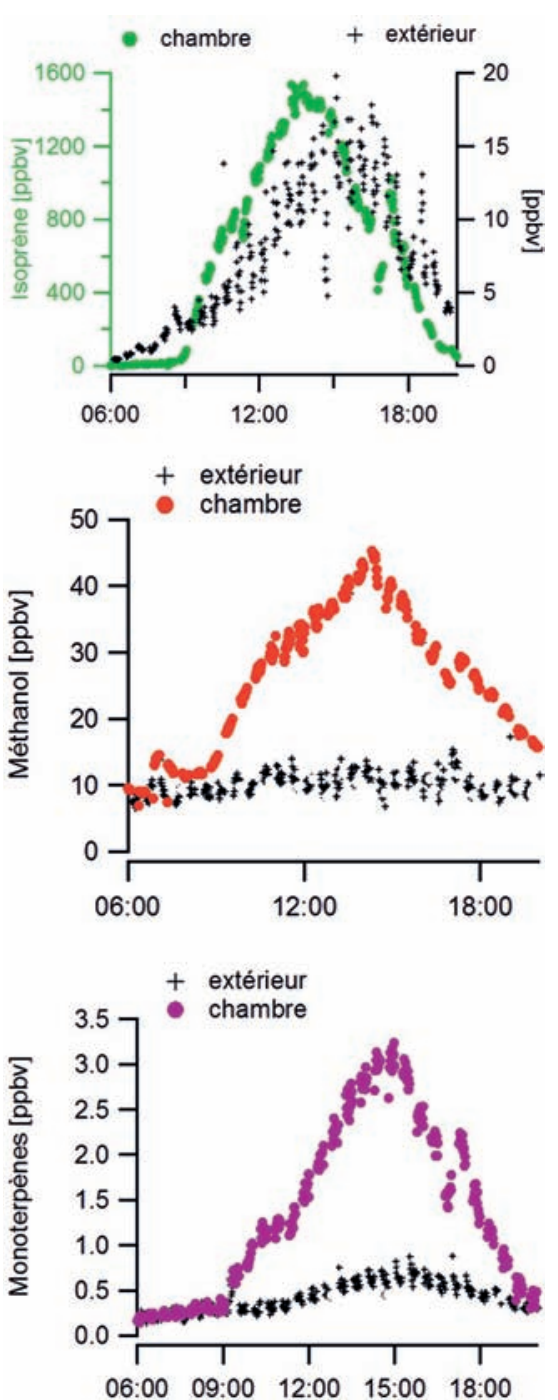

Figure 5. Concentrations en COVB (ppbv) mesurées à l'intérieur et à l'extérieur d'une chambre enfermant une branche de chêne blanc pour trois COVB en fonction de l'heure (locale) de la journée. Expérience réalisée le 1er juin 2012.
L'air échantillonné dans la canopée a été analysé par chromatographie gazeuse (GC-FID) afin de mesurer les hydrocarbures légers (principalement l'isoprène). L'air prélevé au-dessus de la canopée a été analysé par un spectromètre de masse (PTR-MS) pour la mesure rapide de COV ciblés (isoprène, produits de dégradation de l'isoprène, acétone, méthanol, terpènes, acétaldéhyde). Ces mesures couplées à celles de l'anémomètre sonique pour la mesure du vent, ont été utilisées pour le calcul des flux de COV par la méthode de covariance disjointe des turbulences.

\section{Résultats}

\section{Émissions de COVB à l'échelle de la plante}

Le chêne blanc s'est révélé être un fort émetteur d'isoprène, avec un facteur d'émission (taux d'émission normalisés à des conditions de température de $30{ }^{\circ} \mathrm{C}$ et de rayonnement optimal) de $140 \mu \mathrm{g} \mathrm{g}_{\mathrm{sec}}{ }^{-1} \mathrm{~h}^{-1}\left(\mathrm{~g}_{\mathrm{sec}}\right.$ : grammes de biomasse sèche) (Genard-Zielinski et al., 2015). D'autres composés, bien que très minoritaires par rapport à l'isoprène

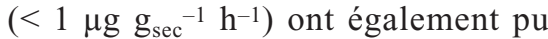
être mesurés, tels que le méthanol, la somme des monoterpènes, l'acétone et l'acétaldéhyde (figure 5). Ce suivi a également révélé un cycle journalier très marqué de ces composés, avec des valeurs quasi nulles durant la nuit, un démarrage des émissions vers 6 heures $\mathrm{du}$ matin et des maxima en milieu de journée (en particulier lors du midi solaire). Cette variabilité est caractéristique des composés biogéniques dont l'émission dépend fortement de la température et du rayonnement solaire.

Les résultats ont montré des facteurs d'émission d'isoprène très variables en fonction de la branche considérée, avec une différence d'un facteur 4 entre la branche la moins émettrice

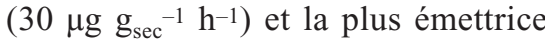

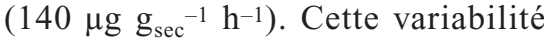
observée pour des arbres de la même espèce est intéressante dans la mesure où elle traduit des différences entre arbres subissant des conditions hydriques, microclimatiques ou physiologiques différentes. Elle met ainsi en évidence la difficulté d'estimer les émissions totales d'une forêt par extrapolation des taux d'émissions mesurés à l'échelle du végétal.

\section{Concentrations et flux de COVB à l'échelle de la canopée}

\section{Suivi saisonnier de l'isoprène}

Un profil saisonnier marqué de l'isoprène a été observé, en lien avec la variation des conditions météorologiques et des caractéristiques phénologiques des arbres. Des concentrations au-dessus des limites de détection ont été observées à partir du mois d'avril, à la poussée des nouvelles feuilles de la chênaie, et ce jusqu'à fin novembre (figure 6). À partir du mois de décembre et jusqu'à la fin de la saison froide, lorsque les feuilles du chêne blanc étaient sèches, les concentrations en isoprène étaient au-dessous des limites de détection.

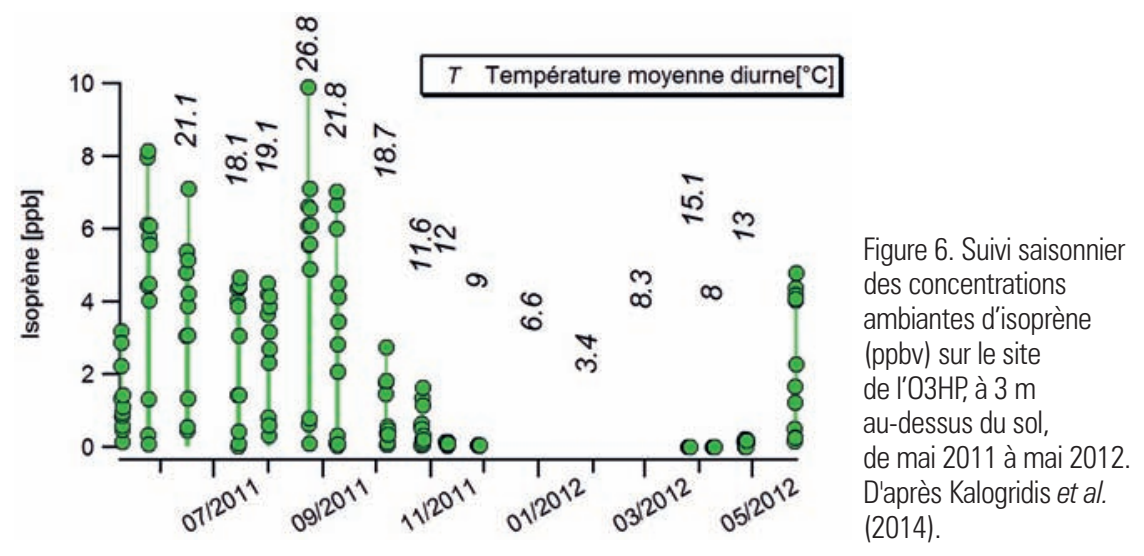




\section{Campagne Canopee (juin 2012)} Dans la forêt de l'O3HP, de fortes concentrations d'isoprène (valeur maximale : $16 \mathrm{ppbv}$ ) et de faibles concentrations de monoterpènes (valeur moyenne : $0,2 \mathrm{ppbv}$ ) ont été observées, ce qui est en accord avec les émissions enregistrées au niveau de la branche du chêne pubescent. Parmi tous les COV, les concentrations d'isoprène présentent la plus grande amplitude entre le jour et la nuit. En présence de lumière, les variations d'isoprène sont plus étroitement liées aux variations de température qu'aux variations d'ensoleillement. Cette corrélation entre les concentrations ambiantes en isoprène et la température de l'air est illustrée sur la figure 7 .

Un gradient dans le profil vertical des concentrations d'isoprène a aussi été observé avec des concentrations plus fortes à l'intérieur de la canopée, près de la source, et des concentrations plus faibles de $40 \%$ au-dessus de la canopée (figure 8).

L'isoprène a montré les plus fortes valeurs de flux sortant de la canopée, avec une émission maximale de $8 \mathrm{mg} \mathrm{m}^{-2} \mathrm{~h}^{-1}$ (figure 9). Une normalisation des valeurs de flux d'isoprène en fonction de la

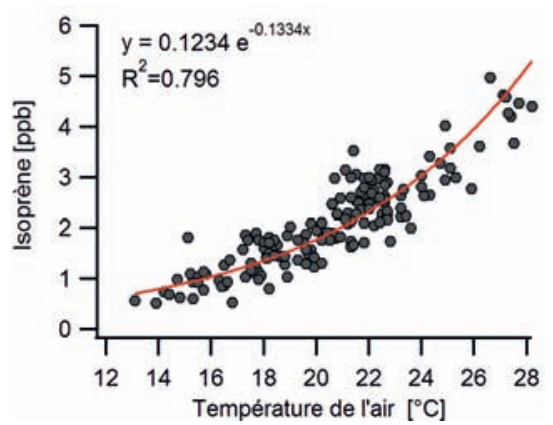

Figure 7. Corrélation entre les concentrations d'isoprène au-dessus du couvert et la température de l'air. température et du rayonnement a permis de dériver un facteur d'émission à l'échelle de la canopée en conditions standard égal à $7,2 \mathrm{mg} \mathrm{m}^{-2} \mathrm{~h}^{-1}$. Les flux mesurés à l'O3HP sont, à notre connaissance, les plus forts jamais enregistrés pour des forêts méditerranéennes, ce qui s'explique aussi en partie par le nombre restreint de mesures de flux ayant eu lieu à ce jour dans le bassin, et ce dans des sites plutôt émetteurs en monoterpènes (Darmais et al., 2000 ; Davison et al., 2009). Ils sont toutefois du même ordre de grandeur que les flux enregistrés dans des écosystèmes très différents, mais fortement émetteurs en isoprène comme certaines forêts des zones tropicales (Amazonie) et tempérées (Allemagne et Belgique). Plusieurs COV oxygénés (méthanol, acétone, acétaldéhyde) ont été mesurés à des concentrations significatives ( $>1$ ppbv) au-dessus de la forêt de l'O3HP, le méthanol étant le plus important et représentant $40 \%$ des concentrations totales de COV mesurés. Le méthanol est le seul composé oxygéné qui ait montré des flux d'émission à l'échelle de la canopée (figure 8). Les flux de méthanol enregistrés sont 5 à 20 fois plus faibles que les flux d'isoprène, alors que les concentrations de méthanol dans l'air ambiant sont supérieures à celles de l'isoprène. Ces fortes concentrations en méthanol s'expliquent par sa longue durée de vie dans l'atmosphère (plusieurs jours contre une heure pour l'isoprène), mais aussi par sa formation à partir d'autres sources, comme la photo-oxydation de précurseurs organiques.

Suite à la quantification des taux d'émission et des niveaux de concentration des COV primaires émis par la végétation, notre étude s'est portée sur les composés secondaires et en particulier sur les produits d'oxydation de l'isoprène. La photo-oxydation de l'isoprène par les radicaux $\mathrm{OH}$ en phase gazeuse conduit à la formation des radicaux hydroxyperoxyles qui peuvent ensuite réagir avec $\mathrm{NO}, \mathrm{RO}_{2}{ }^{5}$ ou $\mathrm{HO}_{2}$ pour finalement former d'autres produits primaires. Les isomères méthylvinylkétone (MVK) et métacroléine (MACR) sont les deux principaux produits de l'oxydation de l'isoprène dans l'atmosphère. Pendant la campagne Canopee, ces deux composés ont présenté une variabilité diurne très proche à celle de l'isoprène (figure 9). Toutefois, les concentrations en MVK et MACR démarrent avec un retard d'environ 2 heures par rapport à l'isoprène, ce qui correspond au temps nécessaire à la photo-oxydation de l'isoprène.

Les niveaux en MVK et MACR au-dessus de la canopée sont inférieurs d'un ordre de grandeur aux concentrations d'isoprène. Le jour, le ratio (MVK + MACR)/isoprène est égal à 0,1 , ce qui est assez bas en comparaison avec d'autres études et témoigne de la faible oxydation de l'isoprène au sein de la forêt. Cette faible oxydation de l'isoprène intracanopée s'explique en partie par les faibles concentrations en oxyde d'azote ( $\mathrm{NO} x$ ). En effet, de nombreuses études cinétiques et de terrain ont montré que dans des conditions de faibles teneurs en NOx l'oxydation de l'isoprène induite par les radicaux hydroxyles $\mathrm{OH}$ conduit à de faibles rendements de MVK et de MACR (Navarro et al., 2011 ; Pinho, 2005 ; Ruppert et Heinz Becker, 2000). La seconde explication est le fait que l'isoprène n'a pas eu le temps de réagir avec les radicaux $\mathrm{OH}$ entre le moment de sa libération par la végétation et son arrivée au point d'échantillonnage (situé

5. $\mathrm{RO}_{2}$ : radical alkylperoxyle, puissant oxydant qui provient de la dégradation de composés organiques sous l'effet du radical hydroxyle $(\mathrm{OH})$ formé à partir de l'ozone.

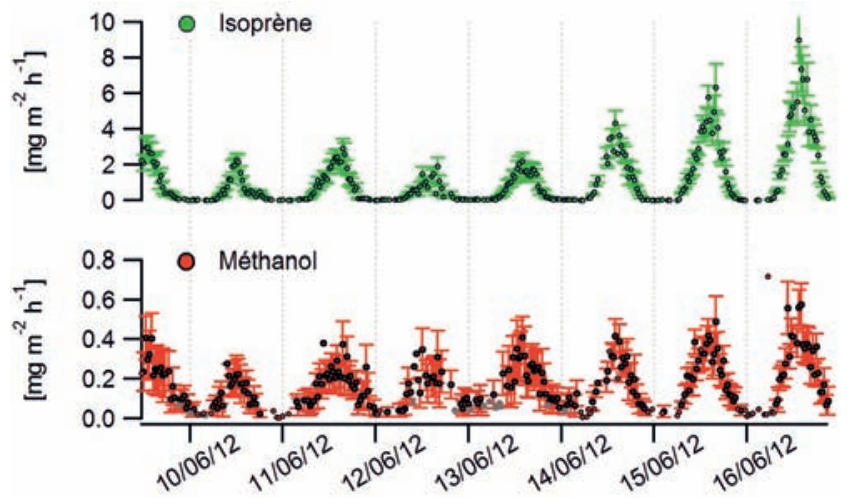

Figure 8. Séries temporelles des flux de COVB (mg m-2 $\left.\mathrm{h}^{-1}\right)$ mesurés au-dessus de la canopée pendant la campagne Canopee (d'après Kalogridis et al., 2014)

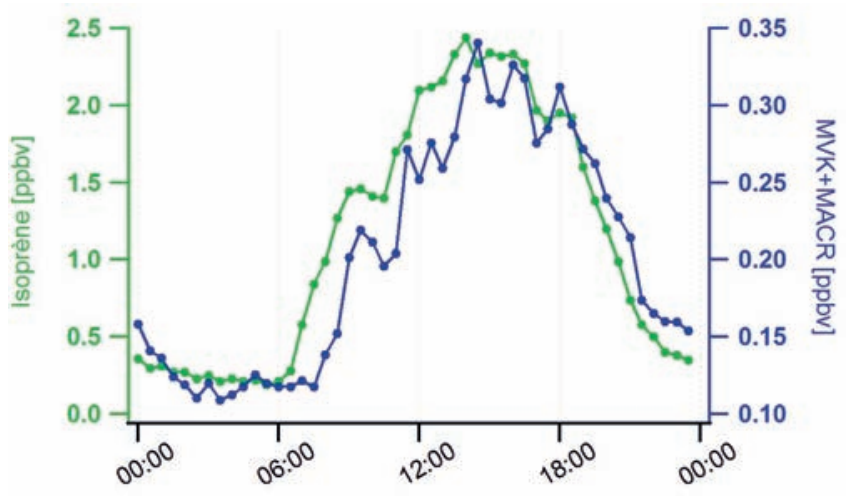

Figure 9. Cycle diurne (moyenné sur toute la période de mesure) des concentrations (ppbv) d'isoprène (en vert) et de ses produits de dégradation (MVK et MACR, en bleu). 
à 10 mètres de hauteur). En effet, comme la canopée O3HP est basse $(5 \mathrm{~m}$ de hauteur) et peu dense, le temps de résidence de l'isoprène au sein de la canopée est trop court. Le temps de transport turbulent entre sol et la hauteur de mesure a été estimé à environ 30-60 s dans la journée, ce qui est bien inférieur au temps nécessaire à l'oxydation de l'isoprène (1-4 h).

De par la structure des forêts méditerranéennes, il semblerait donc que les phénomènes d'oxydation intracanopée ne soient pas significatifs, tout au moins pour des molécules de réactivité similaire (ou inférieure) à l'isoprène.

\section{Contribution des COVB à la formation d'ozone}

L'oxydation photochimique des composés organiques volatils en présence d'oxydes d'azote est une source majeure d'ozone, aussi bien aux échelles locale, régionale que globale. Mais l'efficacité de formation d'ozone est contrôlée par la valeur du rapport $\mathrm{NO} x / \mathrm{COV}$ selon une relation non linéaire. Pour étudier l'impact des émissions de COV sur la formation d'ozone, il est donc nécessaire de déterminer les facteurs limitants (NOx ou $\mathrm{COV}$ ) dans les processus qui mènent à sa formation. Basée sur le diagramme isoplèthe de Seinfeld et Pandis (2006), il apparaît que la production d'ozone à l'OHP est limitée par les oxydes d'azote. Dans ces conditions, le potentiel de formation d'ozone lié aux émissions d'hydrocarbures naturels est négligeable. Toutefois, si les masses d'air chargées en COV sont transportées au-dessus de zones urbaines plus polluées, le rapport COV/NO $x$ va diminuer et les COV présents dans l'air vont contribuer à la formation d'ozone.

Pour déterminer l'impact d'une espèce chimique émise dans l'atmosphère, le point clé consiste à comparer le temps de transport et le temps de résidence atmosphérique de l'espèce. Pour une vitesse moyenne de $8 \mathrm{~m} \mathrm{~s}^{-1}$, le temps nécessaire aux COVB émis à l'OHP pour atteindre le site urbain le plus proche (Forcalquier, situé à une distance de $6 \mathrm{~km}$ ) est estimé à 12 minutes. Basé sur une approche cinétique, il peut être alors estimé que moins de $30 \%$ de l'isoprène a disparu dans cet intervalle de temps. En présence de concentrations de NOx suffisamment élevées, 1 'isoprène pourrait alors contribuer à la formation d'ozone sur le site de Forcalquier. Le temps nécessaire pour atteindre la commune de Manosque est plus long, estimé à 40 minutes. Ce n'est donc qu'une part assez faible des COV émis dans la forêt de l'OHP $(\sim 30 \%$ de 1 'isoprène) qui pourrait atteindre Manosque et réagir avec les NOx pour former de l'ozone. Toutefois, il faut noter que 1'OHP représente un site " idéal », choisi volontairement afin d'être aussi loin que possible de régions urbaines. Cependant, les villes se trouvent souvent entourées de forêts et milieux semi-naturels, émetteurs en COVB. À titre d'exemple, le chêne pubescent occupe une large partie de la commune de Manosque. Située à $5 \mathrm{~km}$ au nord de la ville, la forêt de Pélicier est composée de chênes pubescents, ainsi que de pins noirs d'Autriche, de chênes verts, de pins d'Alep, de pins sylvestres et quelques cèdres. Les niveaux moyens de NOx sont de plusieurs ppbv dans ces régions semi-urbaines ; par conséquent, les émissions de COVB contribuent à la production locale d'ozone. Dans ces conditions et sur la base de l'échelle de réactivité incrémentielle tabulée par Bowman et Seinfeld (1994), ainsi que sur les teneurs de COVB mesurées à l'OHP, le potentiel de formation d'ozone de ces derniers a été estimé approximativement à 8 ppbv (en présence de $\mathrm{NO} x$ ), soit $16 \%$ des concentrations en ozone.

\section{Conclusions et perspectives}

À ce jour, l'estimation des émissions de COVB ainsi que leur devenir chimique sont des sources d'incertitudes majeures. Afin de réduire ces incertitudes, des données consolidées pour différents écosystèmes du monde sont nécessaires. Dans le cadre de la campagne Canopee, la forêt de l'O3HP s'est révélée être un bon site d'étude en raison de sa végétation fortement émettrice en COVB et représentative du bassin méditerranéen, ainsi que de la faible influence des émissions anthropiques. Pendant la campagne de mesure intensive, les teneurs de COVB et les flux d'émission ont été mesurés à plusieurs niveaux : de la branche à la canopée. Les études en chambres ont permis l'étude des émissions à l'échelle du végétal et ainsi la meilleure compréhension des paramètres environnementaux et physiologiques de la plante qui régissent les émissions. Les données de flux au-dessus de la canopée ont permis de quantifier les flux de COVB sortant de la canopée et entrant dans la troposphère. Les résultats montrent que le chêne blanc est un fort émetteur d'isoprène, mais un faible émetteur de monoterpènes et de composés oxygénés. À l'échelle de la forêt, les flux d'émission d'isoprène ont été estimés à $7,2 \mathrm{mg} \mathrm{m}^{-2} \mathrm{~h}^{-1}$ en conditions standard (température de $30{ }^{\circ} \mathrm{C}$ et rayonnement optimal). Néanmoins, l'étude de la composition atmosphérique en termes de concentrations ou de flux d'émission ne permet pas à elle seule d'appréhender l'importance relative de chacun des composés étudiés dans la chimie de l'atmosphère. Ainsi, à partir des indices caractéristiques de la réactivité, il a été montré que les COVB peuvent être des précurseurs importants d'ozone, en particulier dans des sites qui sont simultanément sous l'influence d'émissions anthropiques et biogéniques.

\section{Contribution des auteurs}

La contribution de C. Kalogridis, V. Gros et B. Bonsang a porté sur la quantification des flux de COV à l'échelle de l'écosystème. A.-C. Genard, C. Boissard, C. Fernandez et $\mathrm{E}$. Ormeno ont contribué à ces travaux par la mise en œuvre des mesures et l'étude des émissions de COV à l'échelle du végétal. R. Sarda-Estève, N. Bonnaire et D. Baisnée ont apporté leur soutien technique (sur le terrain et au laboratoire) pour la mise en œuvre des mesures et l'analyse off-line des échantillons prélevés dans la forêt de l'O3HP. Finalement, J. Lathière, a assuré la fonction de coordinatrice de l'ANR Canopee. 


\section{Bibliographie}

Aubinet M., Vesala T., Papale D., 2012. Eddy covariance: a practical guide to measurement and data analysis. Springer.

Chameides W.L., Lindsay R.W., Richardson J., Kiang C.S., 1988. The role of biogenic hydrocarbons in urban photochemical smog: Atlanta as a case study. Science, 241, 1473-1475. doi: 10.1126/science.3420404

Claeys M., Graham B., Vas G., Wang W., Vermeylen R., Pashynska V., Cafmeyer J., Guyon P., Andreae M.O., Artax, P., Maenhaut W., 2004. Formation of secondary organic aerosols through photooxidation of isoprene. Science, 303, 1173-1176. doi: 10.1126/science.1092805

Curci G., Palmer P.., Kurosu T.P., Chance K., Visconti G., 2010. Estimating European volatile organic compound emissions using satellite observations of formaldehyde from the ozone monitoring instrument. Atmos. Chem. Phys., 10, 11501-11517. doi: 10.5194/acp-10-11501-2010

Darmais S., Dutaur L., Larsen B., Cieslik S., Luchetta L., Simon V., Torres L., 2000. Emission fluxes of VOC by orange trees determined by both relaxed eddy accumulation and vertical gradient approaches. Chem. Glob. Change Sci., 2, 47-56. doi: 10.1016/S1465-9972(99)00050-1

Davison B., Taipale R., Langford B., Misztal P., Fares S., Matteucci G., Loreto F., Cape J.N., Rinne J., Hewitt C.N., 2009. Concentrations and fluxes of biogenic volatile organic compounds above a Mediterranean macchia ecosystem in western Italy. Biogeosciences, 6, 1655-1670. doi: 10.5194/bg-6-1655-2009

de Gouw J., Warneke C., 2007. Measurements of volatile organic compounds in the Earth's atmosphere using proton-transfer-reaction mass spectrometry. Mass Spectrom. Rev., 26, 223-257.

Genard-Zielinski A.-C., Boissard C., Fernandez C., Kalogridis C., Lathière J., Gros V., Bonnaire, N., Ormeño, E., 2015. Variability of BVOC emissions from a Mediterranean mixed forest in Southern France with a focus on Quercus pubescens. Atmos. Chem. Phys., 15, 431-446.

Griffin R.J., Cocker D.R., Flagan R.C., Seinfeld J.H., 1999. Organic aerosol formation from the oxidation of biogenic hydrocarbons. J. Geophys. Res., 104, 3555-3567. doi: 10.1029/1998JD100049

Guenther A., Karl T., Harley P., Wiedinmyer C., Palmer P.I., Geron C., 2006. Estimates of global terrestrial isoprene emissions using MEGAN (Model of Emissions of Gases and Aerosols from Nature). Atmos. Chem. Phys., 6, 3181-3210. doi: 10.5194/acp-6-3181-2006

Hauglustaine D.A., Lathière J., Szopa S., Folberth G.A., 2005. Future tropospheric ozone simulated with a climate-chemistry-biosphere model. Geophys. Res. Lett., 32, L24807. doi: 10.1029/2005GL024031

Jacob D.J., Wofsy S.C., 1988. Photochemistry of biogenic emissions over the Amazon forest. J. Geophys. Res., 93, 1477-1486. doi: 10.1029/JD093iD02p01477

Kalogridis C., Gros V., Sarda-Esteve R., Langford B., Loubet B., Bonsang B., Bonnaire N., Nemitz E., Genard A.-C., Boissard C., Fernandez C., Ormeño E., Baisnée D., Reiter I., Lathière J., 2014. Concentrations and fluxes of isoprene and oxygenated VOCs at a French Mediterranean oak forest. Atmos Chem Phys., 14, $10085-10102$. doi:10.5194/acp-14-10085-2014

Karl T.G., Spirig C., Rinne J., Stroud C., Prevost P., Greenberg J., Fall R., Guenther A., 2002. Virtual disjunct eddy covariance measurements of organic compound fluxes from a subalpine forest using proton transfer reaction mass spectrometry. Atmos. Chem. Phys., 2, 279-291. doi: 10.5194/acpd-2-999-2002

Keenan T., Niinemets Ü., Sabate S., Gracia C., Peñuelas J., 2009. Process based inventory of isoprenoid emissions from European forests: model comparisons, current knowledge and uncertainties. Atmos. Chem. Phys., 9, 4053-4076.

Lathière J., Hauglustaine D.A., De Noblet-Ducoudré N., Krinner G., Folberth G.A., 2005. Past and future changes in biogenic volatile organic compound emissions simulated with a global dynamic vegetation model. Geophys. Res. Lett., 32, L20818. doi: 10.1029/2005GL024164

Lee A., Goldstein A.H., Kroll J.H., Ng N.L., Varutbangkul V., Flagan R.C., Seinfeld J.H., 2006. Gas-phase products and secondary aerosol yields from the photooxidation of 16 different terpenes. J. Geophys. Res., 111, 1984-2012.

Navarro M.A., Dusanter S., Hites R.A., Stevens P.S., 2011. Radical dependence of the yields of methacrolein and methyl vinyl ketone from the OH-initiated oxidation of isoprene under NOx-free conditions. Environ. Sci. Technol., 45, 923-929. doi: 10.1021/es103147w

Pinho P.G., Pio C.A., Jenkin M.E., 2005. Evaluation of isoprene degradation in the detailed tropospheric chemical mechanism, MCM v3, using environmental chamber data. Atmos. Environ., 39, 1303-1322. doi: 10.1016/j.atmosenv.2004.11.014

Rinne H.J.I., Guenther A.B., Warneke C., de Gouw J.A., Luxembourg S.L., 2001. Disjunct eddy covariance technique for trace gas flux measurements. Geophys. Res. Lett., 28, 3139-3142. doi: 10.1029/2001GL012900

Ruppert L., Heinz Becker K., 2000. A product study of the OH radical-initiated oxidation of isoprene: formation of C5-unsaturated diols. Atmos. Environ., 34, 1529-1542. doi: 10.1016/S1352-2310(99)00408-2

Seinfeld J.H., Pandis S.N., 2006. Atmospheric chemistry and physics: from air pollution to climate change. Wiley.

Steiner A.H., Goldstein A.L., 2008. Biogenic VOCs. In: Volatile Organic Compounds in the Atmosphere (Koppmann R., ed), John Wiley \& Sons, 82-117.

Taipale R., Ruuskanen T.M., Rinne J., 2010. Lag time determination in DEC measurements with PTR-MS. Atmos. Meas. Tech., 3, 853-862. doi: 10.5194/amt-3-853-2010 